\title{
Wirkungen der verschiedenen Vitamine auf den Glykogenbestand der Leber unter besonders warmer Bedingung.
}

\author{
Von
}

Sinya Sasao.

(䇥生 傎也)

(Aus der Medizinischen Klinik von Prof. T. Kurokawa an der Tohoku Universität zu Sendai.)

(Received for publication, February 6, 1947)

\section{Einleitung.}

Es ist schon von manchen Forschern bekannt gemacht worden, dass das Vitamin $\mathbf{G}$ und die ihm verwandten Stoffe nicht nur antiskorbutisch, sondern auch auf den Kohlehydratstoffwechsel fördernd zu wirken imstande sind; für diesbezügliche nähere Literatur verweise ich auf die schon publizierten Arbeiten aus unserem Institut (Terada, ${ }^{1}$ Simizu, ${ }^{2)}$ Watanabe, ${ }^{\text {s) }}$ Takezawa ${ }^{4)}$ und Miyakosi ${ }^{5}$.

Nach den Arbeiten von Terada ${ }^{1)}$ und Takezawa ${ }^{4)}$ wirken Vitamin C und die ihm verwandten Stoffe (z. B. der rohe Pressaft der verschiedenen Obstarten und verschiedene chemische Stoffe wie 1-Matteucinol, Homoeryodictyol, 7-Oxyflavonon usw.) auf den Glykogenbestand der Kaninchenleber vermehrend und zwar fixierend. In diesem Sinne behält der Obstsaft diese Wirkung auch dann bei, wenn das C-Prinzip von der Hitze zerstört wird. Deshalb sind wir ungezwungen zu dem Schluss gekommen, dass man im Obstsaft noch ein anderes wirksames Prinzip ausser G-Vitamin annehmen muss.

Ich unternehme hier einen Versuch, in dem ich bestimmen will, wie das Vitamin $\mathrm{P}$ bzw. $\mathrm{K}$ im Vergleich mit $\mathrm{G}$ auf den Glykogenbestand der Kaninchenleber im normalen bzw. besonders warmen Zustand wirkt.

\section{Versuchstechnik.}

Um Versuchstiere zu sparen und ausserdem auch zum Zwecke, mit einem und demselben Individuum fortwährend die Veränderung des Glykogenbestandes verfolgen zu können, wendete ich eine neue Methode an, die Prof. Kurokawa und seine Mitarbeiter ${ }^{6)}$ in hiesiger Klinik bear- 
beitet hatten; nämlich ein gesundes Kaninchen, dem vorher die beiderseitigen Intercostalnerven (VIII, IX, X, XI) durchschnitten worden waren, damit das Tier bei der Bauchoperation keine Schmerzen mehr empfinden sollte; wurde laparotomiert und unter der Kontrolle des unbewaffneten Auges von der Leber ein minimales Stückchen mit einer kleinen Klemmschere fast ohne nennenswerte Blutung herausgeschnitten und von diesem Leberstückchen wurde der Gehalt an Glykogen analysiert. Die quantitative Glykogenbestimmung geschah wie folgt : das herausgeschnittene Leberstückchen (ca. $10 \mathrm{mg}$ ) wurde in ein Reagenzglas getan, das ca. $1 \mathrm{ccm} 30$ $\%$ iger Kalilaugelösung enthält und im siedenden Wasserbade vorher erwärmt ist und weiter unter dem Rückflusskühler $30 \mathrm{Min}$. lang bearbeitet. Nach dem Erkalten wurde es mit $10 \%$ iger Salzsäurelösung genau neutralisiert, dann in schwach saurer Lösung (ca. 2\%) 30 Min. lang hydrolisiert, wieder neutralisiert, mit Cadmium-sulfatlösung und normaler Kalilaugelösung enteiweisst. Mit dem Filtrat wurde die reduzierende Kraft analysiert und von dieser der Glykogenwert berechnet. Zu diesem Versuche wurden als Vitamin C Redoxon "Roche" $100 \mathrm{mg}$ pro kg Körpergewicht, als P-Vitamin $5 \mathrm{mg}$ pro $\mathrm{kg}$ Hesperin ,Takeda" und als K-Vitamin $10 \mathrm{mg}$ Katiev ,Takeda" gebraucht.

\section{Versuchsergebnisse.}

Die gesamten Versuche gestalteten sich wie folgt.

A. Bei normalen Kaninchen.

1. Kontrollversuch ohne jegliche Behandlung.

2. Versuch mit alleiniger Anwendung von Glukoselösung.

3. Versuch mit alleiniger Anwendung von Vitaminarten.

4. Versuch mit einer Mischung von Glukose mit Vitamin C.

5. Versuch mit einer Mischung von Glukose mit Vitamin P.

6. Versuch mit einer Mischung von Glukose mit Vitamin $\mathbf{K}$.

7. Versuch mit der kombinierten Anwendung von Glukose mit Vita$\min \mathrm{C}, \mathrm{P}$ und $\mathrm{K}$ zusammen.

B. Unter der Einwirkung hoher Temperatur $\left(42^{\circ} \mathrm{C}\right)$.

1. Kontrollversuch.

2. Versuch mit Glukose allein.

3. Einwirkung der Glukose und des Vitamins C.

4. Einwirkung der Glukose und des Vitamins P.

5. Einwirkung der Glukose und des Vitamins K.

6. Einwirkung der kombinierten Anwendung der Glukose mit Vitamin $\mathrm{C}, \mathrm{P}$ und $\mathrm{K}$ zusammen.

Es ist ziemlich umständlich, diese 13 hintereinander durchgeführten 
Experimente je einzeln zu besprechen; daher habe ich wegen Raumersparnis die Durchschnittszahl der gesamten Versuche in 3 Figuren zusammengestèllt.

Ich habe zuerst die Ergebnisse der Wirkung der alleinigen Anwendung von verschiedenen Vitaminarten auf den Glykogenbestand des normalen Kaninchens in einer Figur zusammengestellt.

In der Figur 1 sind die Minuszahlen unter die Nullinie und die Pluszahlen über diese gestellt. Der Glykogenbestand des Kaninchens, das ohne jegliche Behandlung ruhig im Kasten blieb, vermindert sich in einer Stunde um $0.15 \%$, und es zeigt sich, dass er mit der Zeit etwas abnimmt, aber ohne nennenswerte Schwankungen. Diese Schwankungen sind in den nachfolgenden Angaben immer als Kontrollzahl zum Vergleich in Betracht gezogen werden.

Fig. 1. Wirkung der alleinigen Anwendung der verschiedenen Vitamine auf den Glykogenbestand des normalen Kaninchens.

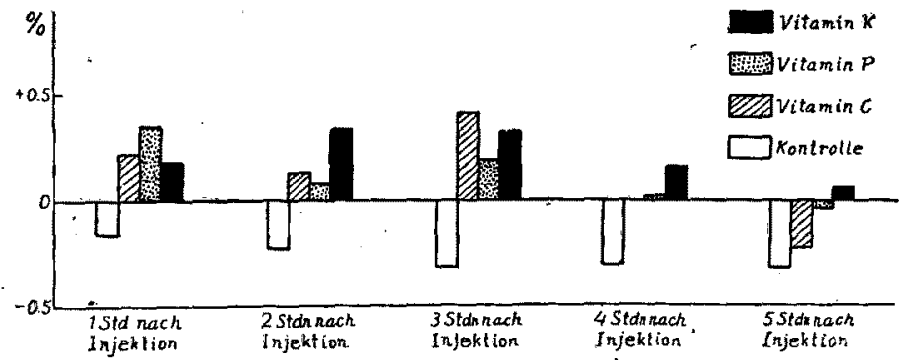

Bei der Anwendung von Vitamin $\mathrm{C}$ allein (100 $\mathrm{mg}$ pro $\mathrm{kg}$ ) wird die Verminderung des Leberglykogens schon 1 Stunde nach der Injektion kompensiert (um 0.22\%), in 2 und 3 Stunden je um 0.13 bzw. $0.42 \%$ wiederhergestellt, und in 5 Stunden ist die Wirkung des C-Vitamins schon vorüber. Bei der Anwendung von Vitamin Pverläuft der Glykogenbestand ziemlich ähnlich wie bei $\mathrm{C}$, aber beim Vitamin $\mathrm{K}$ ist das Leberglykogen im späteren Stadium etwa beibehalten, obwohl im früheren Stadium fast dasselbe Resultat beobachtet wurde.

In der Figur 2 sind die Ergebnisse der Glukose-und Vitaminversuche zusammengestellt. Bei der Darreichung von Glukoselösung allein wird das Leberglykogen schon in einer Stunde ziemlich stark gebildet (etwa $1.40 \%$ ), in 2 Stunden um $1.79 \%$ und in 3 Stunden erreicht es die maximale Höhe, dann vermindert es sich allmählich und zwar in 5 Stunden um etwa $0.66 \%$. Beim Zusatz von Vitamin $\mathrm{C}$ zur Glukoselösung beobachtet man noch mehr die fördernde Wirkung als bei Glukose allein, und zwar erreicht sie in 2 bzw. 3 Stunden die maximale Höhe, dann neigt sie allmählich zur Abnahme. 
Fig. 2. Wirkung der verschiedenen Vitamine auf die Neubildung des Leberglykogens durch intravenöse Infusion der Glukoselösung bei normalen Kaninchen.

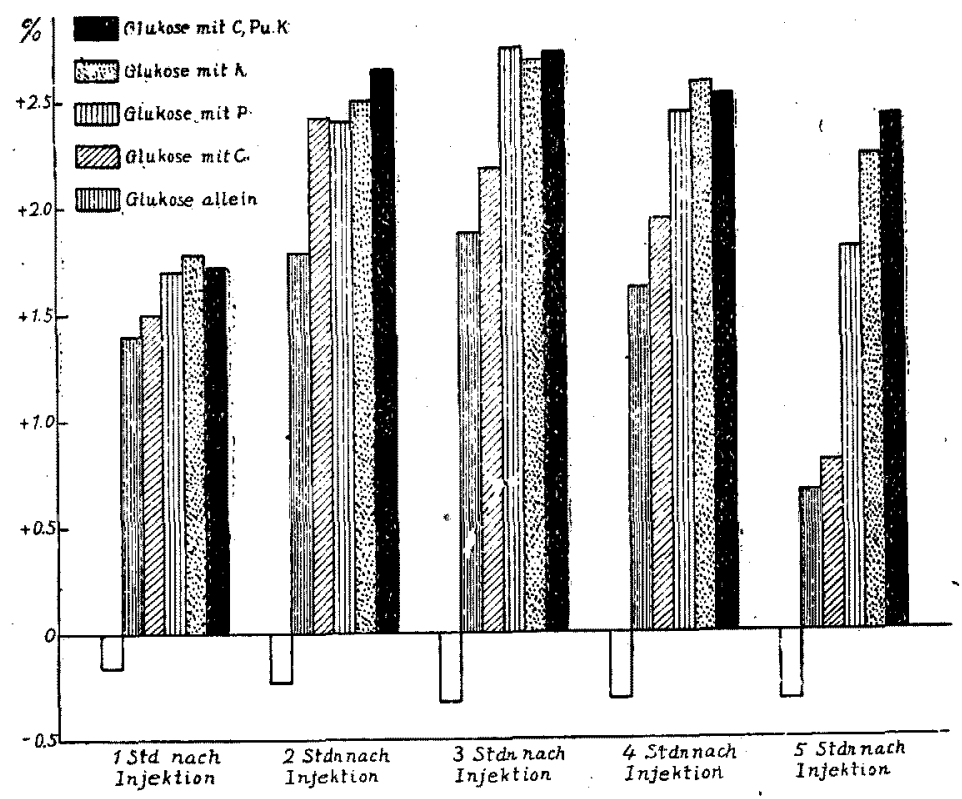

Das Zusetzen des Vitamins $\mathrm{P}$ bzw. $\mathrm{K}$ zur Glukose ruft ebenfalls eine Vermehrung des Glykogens hervor, und zwar in höherem Masse als beim C-Versuch. Charakteristisch ist aber, dass beim P- und K-Versuch die Zersetzung des Glykogens in späteren Stunden ziemlich aufgehalten wird.

Es wird deshalb sehr wahrscheinlich auf die fixierende Wirkung des betreffenden Vitamins zurückgeführt.

Nun ist es das Hauptaugenmerk darauf zu richten, wie sich das Glykogen der Kaninchenleber unter hoher Temperatur verhält und ausserdem wie die genannten Vitaminarten auf die Neubildung des Glykogens aus der Glukoselösung einzuwirken imstande sind.

In der Literatur können wir verschiedene Berichte in bezug auf den Kohlehydratstoffwechsel der Leber unter besonders hoher Temperatur finden $\left(\right.$ Nozaka $^{7}$, Moszeik ${ }^{8}$, Paton ${ }^{9}$, Athanasiu ${ }^{10)}$, Inada ${ }^{11)}$, Iino ${ }^{12)}, \mathrm{Niwa}^{13)}$, Hasegawa ${ }^{14)}$, Imazu ${ }^{15)}$ und Ishimori $\left.{ }^{16)}\right)$.

Obwohl die Versuchstiere und -bedingungen sehr verschieden waren, stimmten die Ergebnisse in der Abnahme des Glykogens fast überein, ausser beim letztgenannten Autor, der förderne Wirkung der Glykogenneubilang beobachtete.

Nun sieht man aus Fig. 3 eine ziemlich hochgradige Zersetztung des Leberglykogens unter Einwirkung hoher Temperatur $\left(42^{\circ} \mathrm{C}\right)$, die besonders ausgeprägt ist in den 5 Stunden nach Einstellung in die Kasten. 
Diese Zersetzung aber wird durch die alleinige Anwendung־der Glukose ziemlich aufgehoben, in einem Masse, dass in 3 Stunden um $1.78 \%$ mehr als der präformierte Wert vorhanden war; doch es kann der Zersetzung durch Wärme nicht so lange trotzen, und nach 5 Stunden findet man nur etwa $0.39 \%$ mehr als zu Anfang.

Fig. 3. Wirkung der verschiedenen Vitamine auf die Neubildung des Leberglykogens durch intravenös infundierte Glukoselösung bei im Kasten $42^{\circ} \mathrm{C}$ erwärmten Kaninchen.

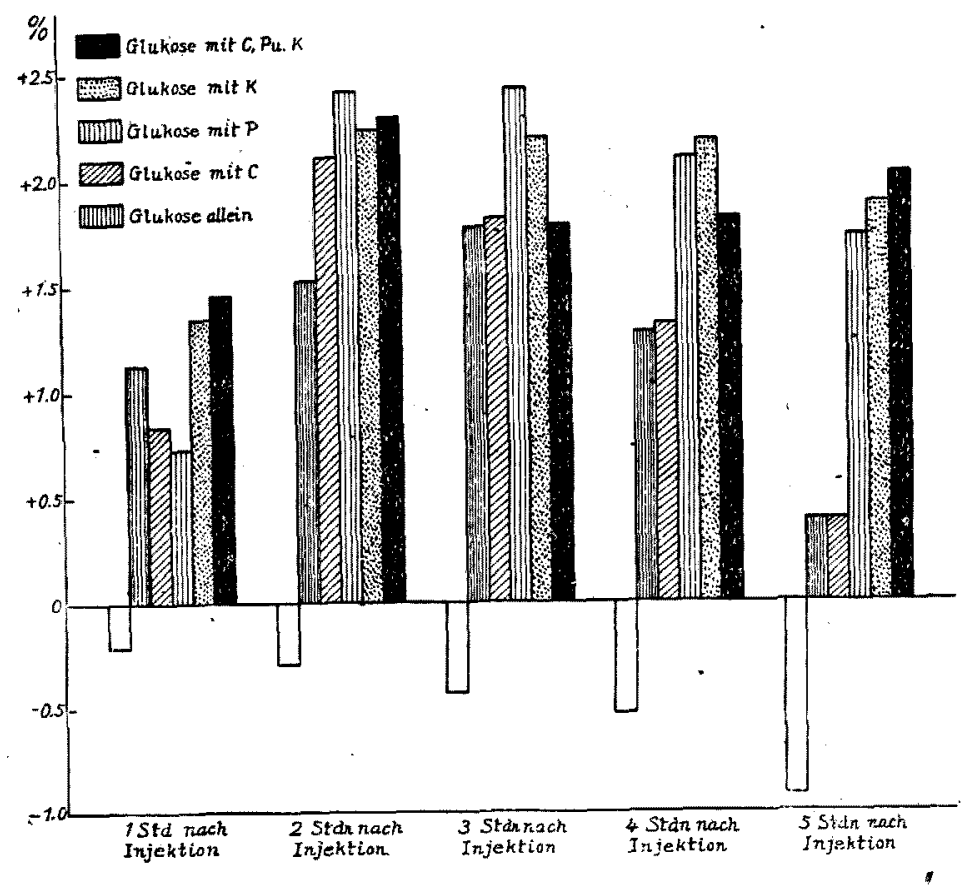

Ich habe zunächst Vitamin C (100 mg pro $\mathrm{kg}$ ) zur Glukoselösung zugesetzt, um zu untersuchen, wie das Leberglykogen sich dazu verhält. Schon 2 Stunden nach der Injektion findet man eine beträchtliche Zunahme des Glykogens, nämlich eine Zunahme um $2.11 \%$, d. h. sie ist um $0.58 \%$ höher als in der Glukose allein, jedoch um $0.31 \%$ geringer als bei demselben Versuch im normalen Zustand, was natürlich als ein Effekt der Wärme zu betrachten ist. Im weiteren Verlauf bis in der fünften Stunde vermindert sich das Leberglykogen allmählich aufs gleiche Niveau wie beim Zucker allein. Also kann man sagen, dass Vitamin $\mathrm{C}$ auf die Neubildung des Glykogens fördernd zu wirken imstande ist, aber man kann ihm wenig fixierende Wirkung zuschreiben.

Im Gegensatz dazu konstatiert man beim Gebrauch von Vitamin P bzw. $K$ nicht nur fördernde, sondern auch fixierende Wirkung des neugebildeten Glykogens. Besonders bei der Anwendung einer Mischung von 
allen 3 Vitaminen in der fünften Stunde findet man mehr Glykogen als in der 3. bzw. 4. Stunde. Es ist besonders hervorzuheben, dass die fixierende Wirkung sehr hervorstechend ist, während sonst unter der Einwirkung vơn Wärme in der 5. Stunde die Zersetzung des Glykogens am stärksten stattfindet.

\section{Schluss.}

Die oben erwähnten Ergebnisse kann man folgenderweise zusammenfassen.

1. Unter besonders warmer Bedingung verliert das Kaninchen das Leberglykogen, was sehr wahrscheinlich zur Annahme berechtigt, dass es vor allen Dingen als Energiequelle mobilisiert wird.

2. In diesem Zustande wird das Leberglykogen durch Glukoseinfusion kompensiert und ausserdem noch Neubildung desselben beobachtet.

3. Der Zusatz von Vitamin $\mathrm{C}, \mathbf{P}$ bzw. $\mathrm{K}$ zur Glukoselösung fördert die Neubildung des Leberglykogens. Úberdies wirkt das Vitamin P bzw. $\mathrm{K}$ auf die Zersetzung des Glykogens fixierend.

4. Es muss wohl warm empfohlen werden, dass man bei warmem Zustande Glukose und Vitaminartèn zusammen verabreichen soll, um das Leberglykogen anzureichern und gleichzeitig den Organismus als ganzen $\mathrm{zu}$ bekräftigen.

\section{Literatur.}

1) Terada, Tohoku Journ. Exp. Med, 1939, 36, 180.

2) Simizu, Ebenda, 1939, 36, 211.

3) Watanabe, Ebenda, 1939, 35, 65.

4) Takezawa, Tohoku Igaku Zasshi, 1940, 26, 15.

5) Miyakosi, im Drucke.

6) Kurokawa und Mitarbeiter, Tohoku Igaku Zasshi, 1943, 33, 437.

7) Nozaka, Nippon Naibumpitsu Gakkai Zasshi, 1929, 3, 1558.

8) Moszeik, Pflüger's Arch., 1888, 42, 556.

9) Paton, zit. nach Senator, Z. kl. Med., 1909, 67, 253.

10) Athanasiu, Pflüger's Arch., 1899, 74, 561.

11) Inada, Tokyo Igakkai Zasshi, 1930, 43, 564.

12) Iino, Kyoto Furitsu Ikadaigaku Zasshi, 1931, 4, 31.

13) Niwa, Ebenda, 1932, 5, 461.

14) Hasegawa, Osaka Igakkai Zasshi, 1936, 34, 1625.

15) Imazu, Journ. Biochem., 1930, 11, 1.

16) Ishimori, Chuo Igakkai Zasshi, 1914, 112, 1. 